\title{
Complex Regional Pain Syndrome Type II After Cervical Interlaminar Epidural Injection
}

Estela Chen Gonzalez, MD, and Vinita Singh, MD

Background: Complex regional pain syndrome (CRPS) is a pain condition associated with autonomic and inflammatory features and is characterized by pain that is disproportionate in magnitude to the typical pain after similar injuries. The pathophysiology of CRPS is poorly understood, and many events have been implicated as causative factors.

Case Report: There are 2 previously documented case reports of CRPS after epidural steroid injections (ESI). This case report details the development of CRPS symptoms in a patient after receiving a cervical ESI. The precipitating event could have been trauma to a nerve root, nerve root irritation from the injectate, or contrast media reaction. Treatment focused on physical therapy and early intervention with a stellate ganglion block. The patient had complete resolution of her symptoms after 10 months.

Conclusions: Although rare, CRPS due to direct nerve root injury or nerve root irritation can develop after an ESl; early diagnosis and treatment may result in a better outcome.

Key words: Complex regional pain syndrome, CRPS, epidural steroid injections, TFESI, ILESI

\section{BACKGROUND}

Epidural steroid injections (ESIs) are usually performed as a conservative approach to the treatment of radicular pain due to disc herniation, spinal stenosis, and other spinal pain syndromes. Complications of ESIs, while rare, do occur and can be related to inadvertent needle placement or adverse reaction to the administered medication. Reported complications of ESIs include infection, hematoma, intravascular injection of medications, dural puncture, air embolism, disc entry, medication reaction, and direct nerve trauma (1). Direct trauma to the nervous tissue can feasibly be the inciting factor for the development of chronic regional pain syndrome (CRPS).

CRPS is a pain condition associated with autonomic and inflammatory features and is characterized by pain that is disproportionate in magnitude or duration to the typical course of pain after similar injuries. CRPS can be subdivided into type I and type II, based on the absence or presence of a known peripheral nerve injury. Despite this distinction, both subtypes are diagnosed clinically based on the International Association for the Study of Pain (IASP) clinical diagnostic criteria for CRPS (Table 1). The pathophysiology of CRPS is poorly understood and is likely multifactorial, including both peripheral and central mechanisms (2).

There are only 2 previously documented case reports of possible CRPS after an ESI $(3,4)$. This case report details the development of symptoms consistent with acute CRPS in a patient after receiving a cervical interlaminar epidural injection for cervical radiculopathy.

From: Department of Anesthesiology, Division of Pain Medicine, Emory University Hospital, 1364 Clifton Rd, Atlanta, GA 30322

Corresponding Author: Estela Chen Gonzalez, MD, E-mail: echengo@emory.edu

Disclaimer: There was no external funding in the preparation of this manuscript.

Conflict of interest: Each author certifies that he or she, or a member of his or her immediate family, has no commercial association (i.e., consultancies, stock ownership, equity interest, patent/licensing arrangements, etc.) that might pose a conflict of interest in connection with the submitted manuscript. Accepted: 2021-03-03, Published: 2021-06-14 


\section{CASE}

A 65-year-old woman with a past medical history of right acoustic neuroma postresection, occipital neuralgia postbilateral occipital and bilateral supraorbital nerve stimulator placement, migraine headaches, myofascial trapezii pain, right temporomandibular joint dysfunction, right greater trochanter pain, major depressive disorder, irritable bowel syndrome, meningioma, a history of shingles, nephrolithiasis, hyperlipidemia, and gastroesophageal reflux disease presented to our chronic pain clinic with a chief concern of cervical neck pain suggestive of cervical radiculopathy. The pain radiated down the right arm in the right $\mathrm{C} 6, \mathrm{C} 7$, and C8 dermatomes. The pain varied between 4-9 out of 10 in severity and was described as throbbing, aching, and tightness. Her cervical computed tomography scan demonstrated multi-level cervical and upper thoracic degenerative changes without significant spinal canal narrowing, as well as moderate right-sided foraminal narrowing at C5-C6 and C7-T1 (Fig. 1). Her pain regimen at the time included baclofen, meloxicam, gabapentin, duloxetine, $5 \%$ topical lidocaine, and physical therapy.

The patient was treated with an elective right paramedian C7 to T1 cervical interlaminar ESI to target the right

Table 1. IASP clinical diagnostic criteria for CRPS.

\begin{tabular}{|c|c|}
\hline \multicolumn{2}{|c|}{ (2) Must report at least one symptom in 3 of the 4 following categories: } \\
\hline Sensory & Reports of hyperesthesia and/or allodynia \\
\hline Vasomotor & $\begin{array}{l}\text { Reports of temperature asymmetry and/or skin } \\
\text { color changes and/or skin color asymmetry }\end{array}$ \\
\hline Sudomotor/edema & $\begin{array}{l}\text { Reports of edema and/or sweating changes and/or } \\
\text { sweating asymmetry }\end{array}$ \\
\hline Motor/trophic & $\begin{array}{l}\text { Reports of decreased range of motion and/or } \\
\text { motor dysfunction (weakness, tremor, dystonia) } \\
\text { and/or trophic changes (hair, nail, skin) }\end{array}$ \\
\hline \multicolumn{2}{|c|}{$\begin{array}{l}\text { (3) Must display at least one sign at time of evaluation in } 2 \text { or more of } \\
\text { the following categories: }\end{array}$} \\
\hline Sensory & $\begin{array}{l}\text { Evidence of hyperalgesia (to pinprick) and/or } \\
\text { allodynia (to light touch and/or deep somatic } \\
\text { pressure and/or joint movement) }\end{array}$ \\
\hline Vasomotor & $\begin{array}{l}\text { Evidence of temperature asymmetry and/or skin } \\
\text { color changes and/or asymmetry }\end{array}$ \\
\hline Sudomotor/edema & $\begin{array}{l}\text { Evidence of edema and/or sweating changes and/ } \\
\text { or sweating asymmetry }\end{array}$ \\
\hline Motor/trophic & $\begin{array}{l}\text { Evidence of decreased range of motion and/or } \\
\text { motor dysfunction (weakness, tremor, dystonia) } \\
\text { and/or trophic changes (hair, nail, skin) }\end{array}$ \\
\hline \multicolumn{2}{|c|}{$\begin{array}{l}\text { (4) There is no other diagnosis that better explains the signs and } \\
\text { symptoms }\end{array}$} \\
\hline
\end{tabular}

C6, C7, and C8 nerve roots. After appropriate monitors were placed and the patient was positioned, prepped, and draped in a sterile fashion, $1 \%$ lidocaine was injected over the entry site using a 27-gauge needle. The C7-T1 interspace was visualized under fluoroscopic imaging. A 20-gauge 3.5-inch needle was inserted and advanced toward the epidural space by means of the "loss-ofresistance" technique to saline. The epidural space was identified, without evidence of blood, cerebrospinal fluid, or paresthesia throughout. A catheter was then advanced to the above level. Placement within the epidural space was confirmed with iohexol injection (Fig. 2), with the epidural space visualized with both anteroposterior and lateral fluoroscopic views. Next, an injectate of $10 \mathrm{mg}$ dexamethasone, one $\mathrm{mL} 1 \%$ lidocaine, and one $\mathrm{mL}$ normal saline was administered into the epidural space. Lastly, the needle was withdrawn. The procedure was well tolerated by the patient, and there were no apparent complications. The preprocedure visual analog pain score was 10 out of 10 . The patient reported $100 \%$ pain relief 15 minutes postinjection. The patient was monitored in recovery for 30 minutes and discharged in stable condition.

Twenty days after the ESI, the patient returned to the pain clinic with recurrent neck pain which started

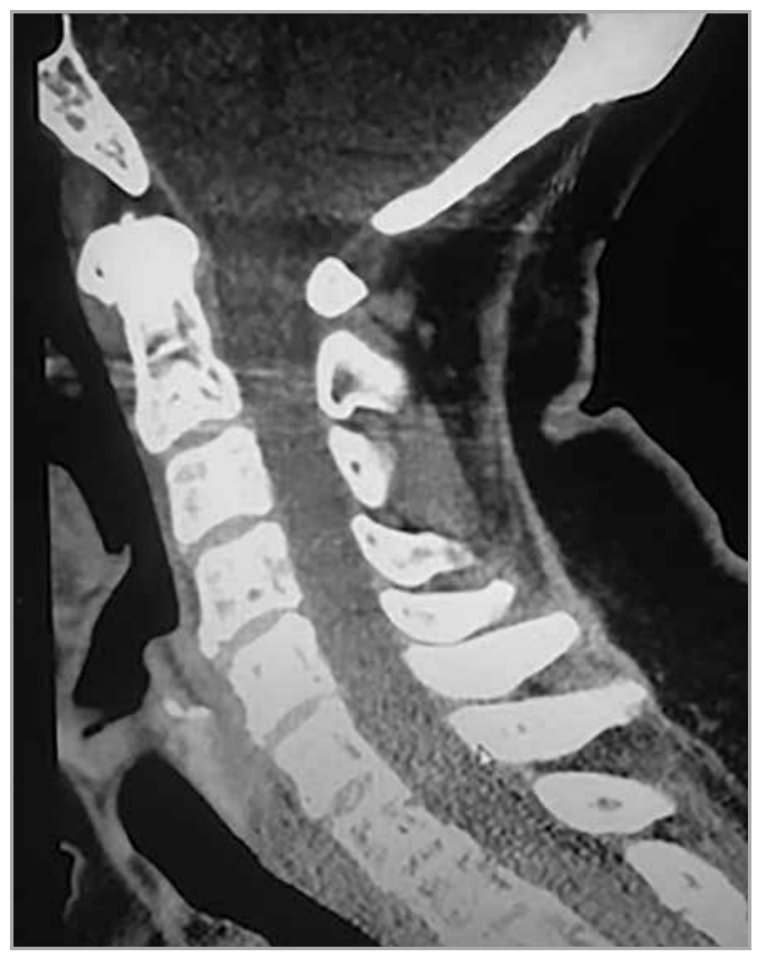

Fig 1. Cervical computed tomography 
on postprocedure day one. The pain was described as 8-10/10 in severity, radiating down the right shoulder into the entire right hand in a nondermatomal pattern, with a throbbing sensation in the proximal part of the arm and pins and needles sensation in the hand, with newly accompanied right-handed weakness and swelling. Her physical exam was notable for mild erythema and swelling of the right hand. The right hand grip strength was $5 / 5$. There was decreased sensation over the thumb in the $\mathrm{C} 6$ distribution on the right compared to the left. There was no allodynia or hyperalgesia.

Given an exam for newly developed CRPS, the patient was started on a clonivdine patch, $0.1 \mathrm{mg}$ per 24 hours for the right hand. One week later, the patient underwent a right-sided level C6 stellate ganglion block under ultrasound guidance for possible CRPS. A 22-gauge $80 \mathrm{~mm}$ Pajunk needle was advanced with in-plane approach under constant ultrasound guidance just deep to the prevertebral fascia into the longus colli muscle (Fig. 3). After negative aspiration, an injectate of $10 \mathrm{~mL}$ of $0.25 \%$ bupivacaine was injected within and above the right longus colli muscle. The block was confirmed by evidence of right-sided Horner syndrome of ptosis, miosis, and differences in skin temperature: $36.2^{\circ} \mathrm{C}$ in the right hand and $35.0^{\circ} \mathrm{C}$ in the left hand. The procedure was well-tolerated by the patient, and there were no apparent complications. The preprocedure visual analog pain score was 10 out of 10 . The patient reported $100 \%$ pain relief 15 minutes postinjection. The patient was monitored in recovery for 30 minutes and discharged in stable condition.

The patient reported several days, up to a week, of relief from the stellate ganglion block. In a follow-up visit 7 weeks later, the pain remained stable. In the interim, she saw a rheumatologist who ruled out rheumatoid arthritis, was started on carbamazepine for neuropathic pain, and continued physical therapy. No additional interventional pain procedures were requested or recommended. Ten months after the ESI procedure, subjective CRPS symptoms, including swelling and weakness, had completely resolved. She continued to have baseline cervical neck pain which was treated using a multimodal pain regimen. This patient was eventually lost to follow-up at our chronic pain clinic due to a diagnosis of stage IV cholangiocarcinoma that was not responsive to chemotherapy. The patient was eventually admitted to home hospice.

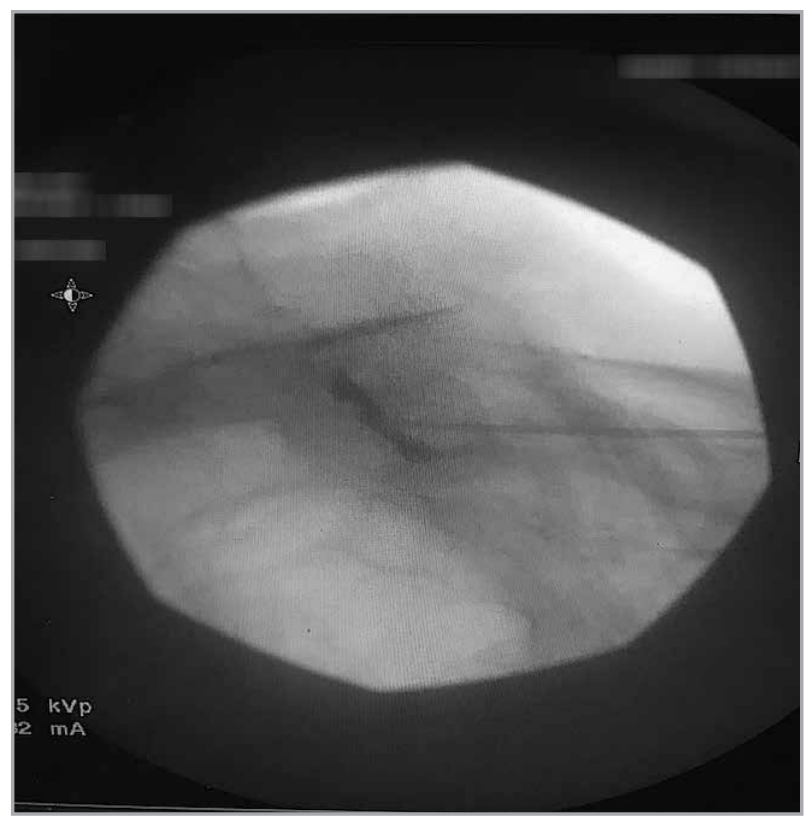

Fig 2. Interlaminar injection (contralateral oblique view)

\section{DISCUSSION}

Complex regional pain syndrome is a disorder of a body region not in a specific nerve root distribution or dermatome, characterized by pain, abnormal sensory, motor, vasomotor, sudomotor, and trophic findings (5). The pain is typically in a distal limb and is often disproportionate in magnitude and duration to the typical course of pain after similar injuries. CRPS is distinguished from other chronic pain syndromes by the presence of autonomic and inflammatory changes in the region of the pain. The affected limb may display changes in skin color and skin temperature, sweating, edema, altered patterns of hair growth, allodynia, hyperalgesia and reduced strength due to pain, edema, or contractures. Some patients develop central motor dysfunction such as tremors, myoclonus, and dystonia. Former names for CRPS in the literature include reflex neurovascular dystrophy, neuroalgodystrophy, shoulder-hand syndrome, reflex sympathetic dystrophy and causalgia (6). CRPS can be subdivided in to type I and type II. CRPS type I corresponds to patients without peripheral nerve injury, and type II corresponds to cases with clinical signs of major peripheral nerve injury, such as nerve conduction study abnormalities. The pathophysiologic significance and clinical utility of the distinction between type I and type II is unclear, as both CRPS subtypes are currently diagnosed and treated similarly. 


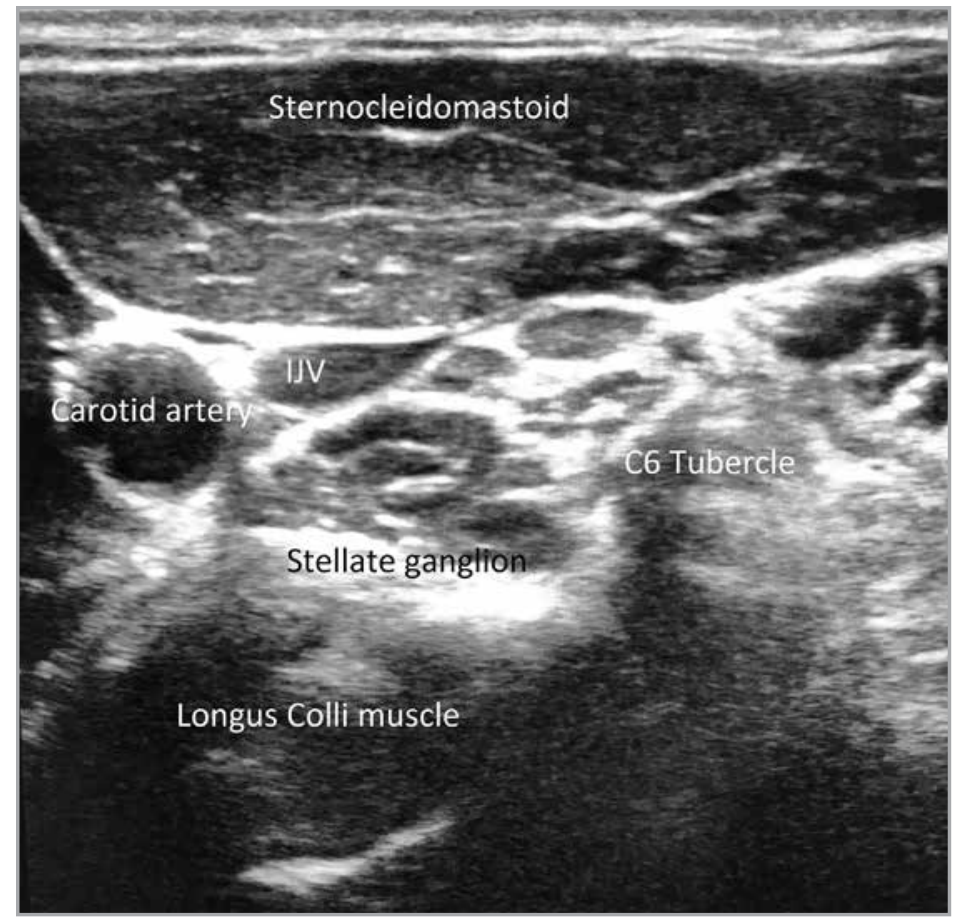

Fig 3. Ultrasound picture of stellate ganglion located on top of longus colli muscle.

$\mathrm{IJV}=$ Internal jugular vein

The incidence of CRPS in the general population is between 5 to 26 per 100,000 per year (7-9). CRPS is more common in women with a female-to-male ratio of 3:1 to $4: 1$, and more commonly affects postmenopausal women. The upper extremities are more frequently affected compared to the lower extremities, and fracture is the most common inciting event (7). Sprains, contusions, crush injuries, surgery, and carpal tunnel syndrome are also known causes (9).

The pathophysiology of CRPS is not well understood. The syndrome is likely a multifactorial process including central and peripheral sensitization, abnormal sympathetic nerve function, inflammatory and immune related factors, genetic factors, and psychological factors. Given the diverse presentation of CRPS and the propensity for symptoms to change over time, the contributions of different mechanisms probably differs between patients and even within the same patient over time (2).

There are 2 previously published case reports of CRPS after a cervical ESI, one after a transforaminal approach and one after an interlaminar approach $(3,4)$. Though the likelihood of nerve root trauma would appear to be higher with the transforaminal approach, nerve root irritation and damage can also be possible with needle placement into the epidural space with the interlaminar approach. Patients in both case reports developed sudden onset pain and paresthesia in the targeted extremity during the procedure, which later developed into a constellation of symptoms consistent with CRPS.

In this case, our patient was diagnosed based on The Budapest Criteria or IASP clinical diagnostic criteria for CRPS. She described continuous right upper extremity pain out of proportion of the inciting event, and had sudomotor (swelling), vasomotor (skin color changes), and motor findings (weakness) of her right hand. Additionally, no other diagnosis better illustrated the above presentation. The precipitating event for the development of CRPS in our patient could have been trauma to a nerve root after an ESI. Severe acute pain would be expected with direct nerve trauma during an ESI. However, our patient did not describe acute pain or paresthesia with needle placement and medication administration. It is possible that she developed nerve root irritation from the injectate, which would be more consistent with the lack of intraprocedure pain and a more delayed pain presentation. Alternative less likely explanations include contrast medium reaction or transient changes in pain tolerance during the procedure. It is highly likely that her presentation was related to the ESI procedure, given the time course of her symptoms, but it is worth noting that up to $10 \%$ of patients with CRPS have no identifiable inciting event (8).

The successful management of CRPS starts with early recognition and a high index of suspicion when patients present with the corresponding constellation of symptoms. The diagnosis is purely clinical, and there is no gold standard testing to confirm the diagnosis. Some have attested the utility of imaging techniques such as 3-phase bone scintigraphy showing increased radiotracer uptake in the affected area and side-by-side radiographs showing blotchy bone decalcification in the affected side (10). Negative imaging does not rule out the diagnosis of CRPS.

A comprehensive and multidisciplinary approach is recommended for the treatment of CRPS, with a 
goal of minimizing pain and disability while restoring function to the affected limb. It appears that early intervention is emphasized and more effective, ideally before there is evidence of radiographic changes (11). Although large cohort randomized controlled trials to guide the treatment of CRPS are still limited, expert consensus on the appropriate initial management include patient education, physical therapy, occupational therapy, and nonopioid analgesics for pain control. As with other forms of chronic pain syndromes, some patients may benefit from psychosocial and behavioral therapy. Nonopioid analgesic options for CRPS include nonsteroidal anti-inflammatory drugs, analgesic antidepressants, and anticonvulsants. Oral corticosteroids may be effective in early CRPS, but is not effective in treating chronic CRPS (2). Patients who are not improving with noninvasive therapy care are candidates for increasingly invasive interventions. Interventional therapies for the treatment of CRPS include trigger point injections, sympathetic nervous system blocks, and spinal cord stimulation for those with refractory pain symptoms.

\section{CONCLUSION}

In conclusion, we report a case of CRPS, likely caused by a cervical nerve root injury following an interlaminar ESI. Our patient had a pre-existing chronic pain syndrome (occipital neuralgia) and was on a nonopioid analgesics regimen prior to the diagnosis of iatrogenic CRPS, which warranted early interventional therapy. Her CRPS symptoms were controlled and eventually resolved 10 months later with a combination of physical therapy, adjunctive pain medications, and sympathetic blockade of the stellate ganglion.

\section{REFERENCES}

1. Boswell MV, Trescot AM, Datta $S$, et al. Interventional techniques: Evidence-based practice guidelines in the management of chronic spinal pain. Pain Physician 2007; 10:7-111.

2. Bruehl S. Complex regional pain syndrome. BMJ 2015; 351:h2730.

3. Park GY, Kwon DR, Kwon DG. Complex regional pain syndrome type II after cervical transforaminal epidural injection: A case report. Medicine (Baltimore) 2018; 97:e10784.

4. Siegfried RN. Development of complex regional pain syndrome after a cervical epidural steroid injection. Anesthesiology 1997; 86:1394-1396

5. Harden RN, Bruehl S, Stanton-Hicks M, Wilson PR. Proposed new diagnostic criteria for complex regional pain syndrome. Pain Med 2007; 8:326-331.

6. Stanton-Hicks M, Jänig W, Hassenbusch S, Haddox JD, Boas R, Wilson P. Reflex sympathetic dystrophy: Changing concepts and

taxonomy. Pain 1995; 63:127-133.

7. Sandroni P, Benrud-Larson LM, McClelland RL, Low PA. Complex regional pain syndrome type I: Incidence and prevalence in Olmsted county, a population-based study. Pain 2003; 103:199-207.

8. de Mos M, de Bruijn AG, Huygen FJ, Dieleman JP, Stricker BH, Sturkenboom MC. The incidence of complex regional pain syndrome: A population-based study. Pain 2007; 129:12-20.

9. Ott $S$, Maihöfner $C$. Signs and symptoms in 1,043 patients with complex regional pain syndrome. J Pain 2018; 19:599.

10. Birklein F, O'Neill D, Schlereth T. Complex regional pain syndrome: An optimistic perspective. Neurology 2015; 84:89-96.

11. Harden RN, Oaklander AL, Burton AW, et al. Complex regional pain syndrome: Practical diagnostic and treatment guidelines, 4th edition. Pain Med 2013; 14:180-229. 
